\title{
ACIDENTE POR SERPENTES DO GÊNERO BOTHROPS: SÉRIE DE 3.139 CASOS
}

\author{
Lindioneza Adriano Ribeiro e Miguel Tanús Jorge
}

\begin{abstract}
Em avaliação dos prontuários médicos de 3.139 pacientes picados por serpentes do gênero Bothrops atendidos no Hospital Vital Brazil (HVB), de 1981 a 1990, observouse maior acometimento do sexo masculino (75, 7\%). Em 1.412 casos (45,0\%) a serpente foi identificada, sendo $1.376 \mathrm{~B}$. jararaca, $20 \mathrm{~B}$. jararacussu, $11 \mathrm{~B}$. neuwiedi, $2 \mathrm{~B}$. moojeni, $2 \mathrm{~B}$. alternatus e $1 \mathrm{~B}$. pradoi. As regiões anatômicas mais comumente picadas foram: pé (47,5\%) e mão (21,3\%). O torniquete foi realizado em 38,2\% dos casos e sua freqüência diminuiu durante esse período $(p<0,001)$. As manifestações clinicas mais freqüentes foram: dor (95,6\%), edema (95,4\%), equimose $(56,1 \%)$, bolha $(13,8 \%)$, necrose (16,5\%), abscesso (11,0\%), sangramento extratecidual (12,3\%), insuficiência renal (1,6\%) e choque (0,7\%). A coagulação sanguínea foi avaliada em 2.990 casos $e$ estava alterada em 1.730 (57,9\%). Ocorreram 21 amputações $(0,7 \%)$ e 9 óbitos $(0,3 \%)$. $A$ dose de soro administrada no HVB diminuiu ao longo desse período $(p<0,001)$.
\end{abstract}

Palavras-chaves: Picada de cobra. Acidente botrópico. Acidente por Bothrops. Envenenamento ofídico.

As serpentes venenosas existentes no Brasil pertencem aos gêneros Bothrops, Crotalus, Lachesis e Micrurus, que são responsáveis por mais de 20.000 acidentes notificados anualmente ao Ministério da Saúde. Aproximadamente 85\% dos envenenamentos são causados por Bothrops 16 17. No Estado de São Paulo não existem Lachesis na natureza e dentre 6.834 casos de acidentes notificados à Secretaria de Saúde de São Paulo em que houve referência ao gênero da serpente (1986 a 1989), espécies de Bothrops foram responsáveis por 85,6\% dos casos 18. Essa percentagem, entretanto, varia com a região do estado 727 . Embora serpentes desse gênero existam em todo o Brasil, as várias espécies distribuem-se irregularmente em seu território6.

O veneno botrópico possui três atividades principais: 1) proteolítica ou necrosante, determina edema inflamatório na região da picada; 2) coagulante, atua através de uma ou mais ações como trombina simile (semelhante à ação da trombina), ativadora de protrombina e do fator X, promovendo consumo dos fatores de coagulação com conseqüente alteração da coagulação sanguínea; 3) hemorrágica, que atua no endotélio vascular na região da picada

Endereço para correspondência: Dr $^{\mathrm{a}}$ Lindioneza Adriano Ribeiro. Dept ${ }^{\circ}$ Clínica Médica/ UFU. Av. Pará 1720, Campus Umuarama, 38400-902 Uberlândia, MG.

Fax: (034) 2182349

Recebido para publicação em 19/02/97. e, provavelmente, também à distância. Outras atividades que podem participar da fisiopatologia do envenenamento são a fibrinogenolítica, fibrinolítica e agregadora de plaquetas 92.

O presente estudo é uma avaliação da epidemiologia do acidente e do quadro clínico do envenenamento por serpentes do gênero Bothrops, com base em uma grande casuística do Hospital Vital Brazil, do Instituto Butantan, São Paulo, SP.

\section{MATERIAL E MÉTODOS}

Os dados do presente estudo foram obtidos dos prontuários médicos dos pacientes picados por serpentes do gênero Bothrops, atendidos no Hospital Vital Brazil do Instituto Butantan (HVB-IB), no período de 1981 a 1990. Devido às características do HVB-IB, que há mais de 50 anos dedica-se ao atendimento dos acidentes ofídicos, os prontuários médicos são dirigidos para os envenenamentos por animais peçonhentos, o que facilita a obtenção de informações precisas. Isso foi possível também porque os dados foram colhidos periodicamente, ao longo dos anos em que os autores trabalharam no HVB-IB e algumas informações foram, na realidade, colhidas prospectivamente ou mesmo checadas após terem sido obtidas dos prontuários.

As serpentes trazidas ao HVB-IB recebiam identificação no Laboratório de Herpetologia. O tempo de coagulação (TC) foi mensurado, à admissão no HVB, através da observação da 
Ribeiro LA, Jorge MT. Acidente por serpentes do gênero Bothrops: série de 3.139 casos. Revista da Sociedade Brasileira de Medicina Tropical 30:475-480, nov-dez, 1997.

coagulação de alguns mililitros de sangue colocados em dois tubos de vidro, geralmente em banho maria a $37^{\circ} \mathrm{C}$, mas às vezes, à temperatura ambiente. Considerava-se normal a coagulação de todo o conteúdo do tubo em até 15 minutos. As análises estatísticas foram realizadas pelo teste do $\chi^{2}$.

\section{RESULTADOS}

Durante o período de 1981 a 1990 foram atendidos no HVB-IB 3.139 pacientes picados por serpentes do gênero Bothrops. Em 1.412 $(45,0 \%)$ casos a serpente foi classificada quanto à espécie. As espécies causadoras dos acidentes foram: B. jararaca (1.376: 97,5\%), B. jararacussu (20: 1,4\%), B. neuwiedi (11: 0,8\%), B. moojeni (2: $0,2 \%), B$. alternatus $(2: 0,2 \%)$ e $B$. prado $i$ (1: $0,1 \%)$. Os acidentes predominaram nos meses de outubro a abril $(83,8 \%)$ (Figura 1), no horário das 12:00 às 18:00 (47,2\%) e das 06:00 às 12:00 (31,3\%), sendo raros das 00:00 às 06:00 horas $(1,4 \%)$. Foram mais acometidas as pessoas do sexo masculino $(75,7 \%)$ e aquelas

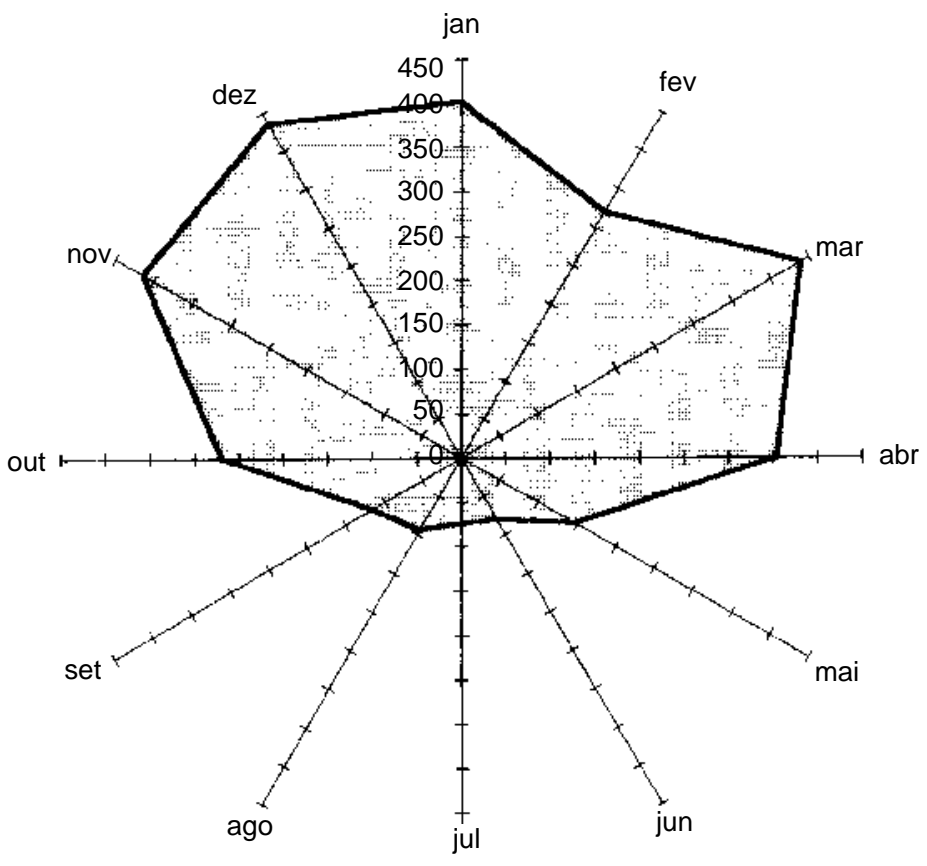

Figura 1 - Sazonalidade do acidente por serpentes do gênero Bothrops, HVB, 1981 a 1990.

na faixa etária de 10 a 40 anos de idade $(59,0 \%)$ (Tabela 1). Quanto à região anatômica acometida $2.342(74,7 \%)$ pacientes foram picados nos membros inferiores, 766 (24,4\%) nos membros superiores, $17(0,5 \%)$ na cabeça e $11(0,4 \%)$ no tronco. Nos membros inferiores foram acometidos o pé $(1.491: 47,5 \%$ dos casos), o tornozelo (390: 12,4\%), a perna (418: $13,3 \%)$ e o joelho ou coxa $(45: 1,4 \%)$ e nos membros superiores as mãos (670:21,3\%), o antebraço $(75: 2,4 \%)$ e o cotovelo ou braço $(22: 0,7 \%)$. O torniquete foi realizado de diferentes formas, intensidades e períodos de tempo, em 1.199 pacientes $(38,2 \%)$. Essa prática foi menos comum nos últimos anos ( $\mathrm{p}<0,001)$ (Tabela $2)$. A maioria $(78,0 \%)$ dos pacientes foi atendida no HVB-IB dentro de 6 horas após a picada (Tabela 3). As manifestações clínicas sistêmicas foram: hemorragia (386 casos: $12,3 \%)$; insuficiência renal $(49: 1,6 \%)$ e choque $(23: 0,7 \%)$. Na região da picada ocorreram dor (3.001:95,6\%), edema (2.994:95,4\%), equimose (1.762:56,1\%), bolha $(434: 13,8 \%)$, necrose $(517: 16,5 \%)$ e abscesso $(346: 11,0 \%)$. Os sangramentos fora da região anatômica picada, excetuando-se, por não ter sido possível conhecer-lhes a localização, as equimoses, apresentaram-se como: gengivorragia (281: 
Ribeiro LA, Jorge MT. Acidente por serpentes do gênero Bothrops: série de 3.139 casos. Revista da Sociedade Brasileira de Medicina Tropical 30:475-480, nov-dez, 1997.

73,0\% dos casos), hematúria $(32: 8,3 \%)$, hematoma (19:4,9\%), hemorragia por ferimentos de pele $(16: 4,2 \%)$, epistaxe $(12: 3,1 \%)$, hematêmese (10:2,6\%), petéquias $(10: 2,6 \%)$ e hemorragia por via genital $(5: 1,3 \%)$. A coagulação sanguínea foi avaliada em 2.990 casos e foi detectado prolongamento do tempo de coagulação em $1.730(57,9 \%)$ sendo que $1.397(46,7 \%)$ pacientes apresentaram incoagulabilidade sanguínea.

Tabela 1 - Sexo e faixa etária dos pacientes picados por Bothrops, HVB-IB, 1981 a 1990.

\begin{tabular}{|c|c|c|c|c|c|c|}
\hline \multirow{3}{*}{$\begin{array}{l}\text { Faixa } \\
\text { etária }\end{array}$} & \multicolumn{6}{|c|}{ Sexo } \\
\hline & \multicolumn{2}{|c|}{ masculino } & \multicolumn{2}{|c|}{ feminino } & \multicolumn{2}{|c|}{ total } \\
\hline & $\mathrm{n}^{\mathrm{O}}$ & $\%$ & $\mathrm{n}^{\mathrm{O}}$ & $\%$ & $\mathrm{n}^{\mathrm{o}}$ & $\%$ \\
\hline$\overline{00+10}$ & 254 & 64,8 & 138 & 35,2 & 392 & 100,0 \\
\hline $10+20$ & 637 & 77,9 & 181 & 22,1 & 818 & 100,0 \\
\hline $20+30$ & 462 & 80,8 & 110 & 19,2 & 572 & 100,0 \\
\hline $30+40$ & 366 & 79,4 & 95 & 20,6 & 461 & 100,0 \\
\hline $40+50$ & 283 & 74,7 & 96 & 25,3 & 379 & 100,0 \\
\hline $50+60$ & 208 & 72,7 & 78 & 27,3 & 286 & 100,0 \\
\hline$>60$ & 164 & 71,0 & 67 & 29,0 & 231 & 100,0 \\
\hline
\end{tabular}

Tabela 2 - Freqüência do uso de torniquete pelos pacientes picados por Bothrops segundo o ano de ocorrência do acidente, HVB-IB, por Bothrops

\begin{tabular}{|c|c|c|c|c|c|c|}
\hline \multirow{3}{*}{ Ano } & \multicolumn{6}{|c|}{ Uso de torniquete } \\
\hline & \multicolumn{2}{|c|}{ sim } & \multicolumn{2}{|c|}{ não } & \multicolumn{2}{|c|}{ total } \\
\hline & $\mathrm{n}^{\mathrm{O}}$ & $\%$ & $\mathrm{n}^{\mathrm{O}}$ & $\%$ & $\mathrm{n}^{\mathrm{O}}$ & $\%$ \\
\hline 1981 & 99 & 39,4 & 152 & 60,6 & 251 & 100,0 \\
\hline 1982 & 128 & 41,5 & 180 & 58,5 & 308 & 100,0 \\
\hline 1983 & 104 & 44,4 & 130 & 55,6 & 234 & 100,0 \\
\hline 1984 & 137 & 44,6 & 170 & 55,4 & 307 & 100,0 \\
\hline 1985 & 204 & 43,0 & 270 & 57,0 & 474 & 100,0 \\
\hline 1986 & 153 & 36,9 & 262 & 63,1 & 415 & 100,0 \\
\hline 1987 & 144 & 38,0 & 235 & 62,0 & 379 & 100,0 \\
\hline 1988 & 103 & 33,3 & 206 & 66,7 & 309 & 100,0 \\
\hline 1989 & 62 & 23,7 & 200 & 76,3 & 262 & 100,0 \\
\hline 1990 & 65 & 32,5 & 135 & 67,5 & 200 & 100,0 \\
\hline Total & 1199 & 38,2 & 1940 & 61,8 & 3139 & 100,0 \\
\hline
\end{tabular}

$\mathrm{p}<0,001$.

Tabela 3 - Intervalo de tempo entre a picada por serpente Bothrops Tabela 3 - Intervalo de tempo entre a picada
e o atendimento no HVB-IB, 1981 a 1990.

\begin{tabular}{cccc}
\hline Intervalo de tempo & $\mathrm{n}^{\mathrm{o}}$ & $\%$ & $\%$ acumulada \\
\hline$<01$ & 179 & 5,7 & 5,7 \\
$01+03$ & 1.378 & 43,9 & 49,6 \\
$03+06$ & 892 & 28,4 & 78,0 \\
$06+12$ & 288 & 9,2 & 87,2 \\
$12+24$ & 152 & 4,9 & 92,1 \\
$>24$ & 248 & 7,9 & 100,0 \\
\hline Total & 3.137 & 100,0 &
\end{tabular}
Em dois casos não foi possível obter informação quanto a esse

No HVB-IB o antiveneno foi administrado em $2.758(87,9 \%)$ pacientes, geralmente por via endovenosa, sendo que a grande maioria $(83,9 \%)$ recebeu menos de 10 ampolas de soro antibotrópico poliespecífico (Instituto Butantan).
Houve inicialmente um aumento da dose de soro em 1983/84 e, após, uma redução acentuada $(\mathrm{p}<0,001)$, até os últimos anos estudados (Tabela 4). Vinte e três pacientes $(0,7 \%)$ foram dializados, $21(0,7 \%)$ foram submetidos a amputação de seguimento do membro picado e $9(0,3 \%)$ faleceram. O período de internação variou de um a 90 dias (mediana de 2 dias) sendo que 864 (27,9\%) pacientes permaneceram menos de um dia internados, $1.723(55,7 \%) 1$ ou 2 dias, $334(10,8 \%) 3$ ou 4 dias e $170(5,5 \%)$ 5 ou mais dias. Quarenta e oito pacientes não permaneceram internados no HVB-IB.

Tabela 4 - dose de soro administrada aos pacientes picados por Bothrops segundo o ano em que ocorreu o acidente, HVB, 1981 a 1990.

\begin{tabular}{|c|c|c|c|c|c|c|c|c|}
\hline \multirow{3}{*}{ Ano } & \multicolumn{8}{|c|}{ Dose de soro (número de ampolas) } \\
\hline & \multicolumn{2}{|c|}{1 a 4} & \multicolumn{2}{|c|}{5 a 9} & \multicolumn{2}{|c|}{$10 \mathrm{e}+$} & \multicolumn{2}{|c|}{ Total } \\
\hline & $\mathrm{n}^{\mathrm{O}}$ & $\%$ & $\mathrm{n}^{\mathrm{Q}}$ & $\%$ & $\mathrm{n}^{\mathrm{O}}$ & $\%$ & $\mathrm{n}^{\mathrm{O}}$ & $\%$ \\
\hline$\overline{1981}$ & 45 & 19,8 & 147 & 64,8 & 35 & 15,4 & 227 & 100,0 \\
\hline 1982 & 54 & 19,3 & 179 & 63,9 & 47 & 16,8 & 280 & 100,0 \\
\hline 1983 & 24 & 11,7 & 89 & 43,2 & 93 & 45,1 & 206 & 100,0 \\
\hline 1984 & 38 & 14,3 & 138 & 52,1 & 89 & 33,6 & 265 & 100,0 \\
\hline 1985 & 149 & 34,7 & 196 & 45,6 & 85 & 19,8 & 430 & 100,0 \\
\hline 1986 & 165 & 46,1 & 170 & 47,5 & 23 & 6,4 & 358 & 100,0 \\
\hline 1987 & 130 & 39,8 & 175 & 53,5 & 22 & 6,7 & 327 & 100,0 \\
\hline 1988 & 140 & 52,2 & 101 & 37,7 & 27 & 10,1 & 268 & 100,0 \\
\hline 1989 & 140 & 61,8 & 79 & 34,8 & 8 & 3,5 & 227 & 100,0 \\
\hline 1990 & 102 & 60,0 & 54 & 31,8 & 14 & 8,2 & 170 & 100,0 \\
\hline Total & 987 & & 1328 & & 443 & & $2758^{*}$ & \\
\hline
\end{tabular}

\section{DISCUSSÃO}

A freqüência com que diferentes espécies do gênero Bothrops causam os acidentes, conforme atendimento do HVB-IB, não sofreu modificação substancial desde o período de 1954 a 196520 até o atual. A espécie $B$. jararaca, responsável pela maioria dos casos estudados, é de ocorrência em áreas anteriormente ocupadas pela Mata Atlântica, tendo se adaptado às modificações feitas pelo homem ${ }^{24}$, podendo ser encontrada até em áreas verdes da zona urbana da cidade de São Paulo15 24 .

A maior freqüência dos acidentes ocorreu nos meses nos quais, na região estudada, o clima é quente e chuvoso (outubro a abril). Esse período também corresponde ao de maior atividade de $B$. jararaca23. A sazonalidade do acidente, entretanto, parece depender mais das condições ambientais e da atividade do homem. É semelhante para os acidentes por 
Ribeiro LA, Jorge MT. Acidente por serpentes do gênero Bothrops: série de 3.139 casos. Revista da Sociedade Brasileira de Medicina Tropical 30:475-480, nov-dez, 1997.

Crotalus durissus atendidos no mesmo hospital10 e para os acidentes ofídicos em geral, em toda a região sul, sudeste e centrooeste e é diferente para serpentes do mesmo gênero em algumas regiões do país16.

A predominância dos acidentes no período da tarde é concordante com a literatura nacional10 1719 26. Como, no Brasil, a maioria das serpentes peçonhentas tem hábitos vespertinos e noturnos 121 , essa distribuição deve-se, mais provavelmente, à maior atividade diurna do homem do campo.

A maior ocorrência de acidentes com pessoas do sexo masculino tem sido referida em todas as casuísticas nacionais e provavelmente devese à maior freqüência com que os homens realizam atividades no campo. Já foi referida também a acentuada freqüência de acidentes com indivíduos entre 10 ou 15 e 49 ou 50 anos de idade2 5716192529 .

A grande percentagem de picadas nas extremidades inferiores, principalmente nos pés e tornozelos e nas superiores, principalmente nas mãos, já foi anteriormente observada em estudos baseados em acidentes pelas diversas serpentes solenóglifas3 12181920 29. Em 30.037 acidentes notificados de junho de 1986 a dezembro de 1987 ao Ministério da Saúde do Brasil, 58,0\% das picadas aconteceram nos pés ou pernas, portanto, abaixo do joelho16. Isso deve-se aos hábitos terrestres das serpentes peçonhentas brasileiras e à capacidade que têm de desferir bote defensivo até uma distância que não costuma exceder um terço do seu comprimento21.

A freqüência de utilização de torniquete em $38,2 \%$ dos pacientes é grande, uma vez que tem sido desaconselhada4 9 13. Segundo dados do presente estudo, entretanto, tem diminuido nos últimos anos. Isso deve-se, provavelmente ao trabalho de treinamento de profissionais dos diferentes pontos estratégicos para o atendimento de acidentes por animais peçonhentos, implementados principalmente nos últimos anos pela Comissão Permanente de Coordenação e Controle dos Acidentes por Animais Peçonhentos.

O tempo entre o acidente e a administração do soro tem sido considerado importante no prognóstico do envenenamento ofídico. No HVB-IB o tempo entre o acidente e o atendimento foi curto em comparação com o encontrado em outros centros 2830 , exceto pela região de

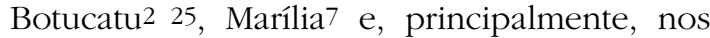
Postos Antiofídicos de Juiz de Fora, onde 63\% dos pacientes foram atendidos dentro de 3 horas 14 . Nesse caso, entretanto, o atendimento se deu de forma descentralizada e, no presente trabalho, não se levou em conta o atendimento antes de chegar ao HVB-IB, mesmo que tenha havido administração de antiveneno.

Embora as manifestações do envenenamento tenham sido as mesmas, a freqüência com que ocorreram foi maior do que a encontrada nos envenenamentos por $B$. jararaca atendidos no próprio HVB-IB, no período de 1981 a 198719. Diferiu dessa casuística também pela ocorrência de casos de óbitos que não foram registrados nos acidentes por B. jararaca19.

A predominância de pacientes que receberam 5 ou mais ampolas de soro $(83,9 \%)$ deve-se ao fato da dose recomendada nesse período para casos moderados e graves ser de 8 e 12 ou mais ampolas, respectivamente13. Embora, em média, tenha havido um aumento na dose de soro administrada nos primeiros anos do estudo, houve diminuição nos últimos anos. Isso deveu-se em parte à deficiência de abastecimento de soro, mas também, pelo menos nos últimos anos, a que estudos recentes, realizados no próprio $\mathrm{HVB}$, sugeriram que as doses preconizadas são exageradas, pelo menos nos casos de envenenamentos leves e moderados por $B$. jararaca 9 11, o que foi posteriormente confirmado 8 . Não pode ser descartada a eventual diminuição gradativa da gravidade dos envenenamentos nos pacientes atendidos durante o período de estudo, embora não haja motivo para se pensar na sua ocorrência.

A percentagem de casos que evolucionaram para o óbito $(0,3 \%)$ revela a pequena gravidade do envenenamento botrópico em área de prevalência de $B$. jararaca, serpente que raramente causa a morte de suas vítimas 19 A letalidade foi a mesma recentemente encontrada para o Estado de São Paulo $(0,3 \%) 18$. Segundo dados da notificação do acidente em todo o Brasil, a letalidade por picada de Bothrops é de aproximadamente $0,5 \% 16$.

O pequeno período de internação na maioria dos casos, deve-se ao fato dos envenenamentos em geral não evoluírem com complicações.

Embora o óbito seja a conseqüência mais temida a amputação de extremidade ocorre 
Ribeiro LA, Jorge MT. Acidente por serpentes do gênero Bothrops: série de 3.139 casos. Revista da Sociedade Brasileira de Medicina Tropical 30:475-480, nov-dez, 1997.

com freqüência mais de duas vezes maior e é também um evento bastante indesejável.

\section{SUMMARY}

Medical records of 3,139 patients bitten by Bothrops snakes and attended at Vital Brazil Hospital (HVB) from 1981 to 1990 were reviwed. They were more frequent in males (75.7\%). In 1,412 cases (45.0\%) the snake was classified by species, and 1,376 were $\mathrm{B}$. jararaca, $20 \mathrm{~B}$. jararacussu, $11 \mathrm{~B}$. neuwiedi, $2 \mathrm{~B}$. moojeni, $2 \mathrm{~B}$. alternatus $e 1 \mathrm{~B}$. pradoi. The most frequent bitten anatomic regions were: foot (47.5\%) and hand (21.3\%). Tourniquet was used in 38.2\% of the cases and its frequency fell down during the study period $(p<0.05)$. The clinical features at the bite site were: pain (95.6\%), swelling (95.4\%), echimosis (56.1\%), blisters (13.8\%), necrosis (16.5\%), and abscess (11.0\%). Systemic manifestations were: bleeding (12.3\%), acute renal failure (1.6\%), and shock (0.7\%). There were blood coagulation disorders in 1, 730 (57.9\%) of the 2,990 cases. There were 21 amputations (0.7\%) and 9 deaths (0.3\%). The average serum dose that was used in treatment fell down during the study period $(p<0.001)$.

Key-words: Snake bite. Bothropic accident. Bothrops. Ophidic envenoming.

\section{AGRADECIMENTOS}

À equipe de médicos e demais funcionários do Hospital Vital Brazil do Instituto Butantan, que, durante todo esse período, prestou atendimento aos pacientes.

\section{REFERÊNCIAS BIBLIOGRÁFICAS}

1. Amaral A. Contribuição à biologia dos ophidios brasileiros (habitat, hábitos e alimentação). Coletâneas de Trabalhos do Instituto Butantan (1918-1924) 2:177-181, 1927.

2. Barravieira B, Pereira PCM. Acidentes por serpentes do gênero Bothrops, Lachesis e Micrurus. Arquivos Brasileiros de Medicina 65:345-355, 1991.

3. Barroso RD. Ofidismo no Brasil: considerações em torno de 2.238 acidentes ofídicos tratados com soro. Boletim do Instituto Vital Brazil 26:35-47, 1944.

4. Brasil, Ministério da Saúde. Secretaria Nacional de Ações Básicas de Saúde. Ação do Ministério da Saúde no controle dos acidentes por animais peçonhentos. Brasília. 1988.
5. Brazil V. A defesa contra o ophidismo. Pocai \& Weiss, São Paulo, 1911.

6. Campbell JA, Lamar WW. The venomous reptiles of Latin America. Cornell University Press, Ithaca, 1989.

7. Garcia FCM, Fulini DR, Mendes RP, Barravieira B, Marcondes-Machado J, Pereira PCM, Souza LR, Zornof DCM, Meira DA. Estudo clínicoepidemiológico de doentes picados por serpentes venenosas, na região de Botucatu (SP). Jornal Brasileiro de Medicina 67:224-232, 1994.

8. Jorge MT, Cardoso JLC, Castro SCB, Ribeiro LA, França FOS, Sbrogio de Almeida ME, Kamiguti AS, Sano-Martins IS, Santoro ML, Mancau JEC, Warrell, DA, Theakston RDG. A randomized 'blinded' comparison of two doses of antivenom in the treatment of Bothrops envenoming in São Paulo, Brazil. Transactions of The Royal Society of Tropical Medicine and Hygiene 89:111-114, 1995.

9. Jorge MT, Ribeiro LA. Acidentes por serpentes peçonhentas do Brasil. Revista da Associação Médica Brasileira 36:66-77, 1990.

10. Jorge MT, Ribeiro LA. Epidemiologia e quadro clínico do acidente por cascavel sul-americana (Crotalus durissus). Revista do Instituto de Medicina Tropical de São Paulo 34:347-354, 1992.

11. Jorge MT, Ribeiro LA. Efeito da redução na dose de soro antibotrópico administrada a pacientes picados por serpentes do gênero Bothrops. Revista da Associação Médica Brasileira 40:59-62, 1994.

12. Kouyoumdjian JA, Polizeli C. Acidente ofídico causado por Bothrops moojeni: relato de 37 casos. Revista do Instituto de Medicina Tropical de São Paulo 30:424-432, 1988.

13. Manual para o diagnóstico e tratamento de acidentes por animais peçonhentos. São Paulo, 1982.

14. Mourão ELS. Aspectos sociais do acidente ofídico na arquidiocese de Juiz de Fora. Cave, Juiz de Fora, 1971.

15. Puorto J, Sazima I, Laporta-Ferreira IL. Serpentes da região urbana de São Paulo, In: Resumos do XVII Congresso Brasileiro de Zoologia, Londrina p. 442, 1990.

16. Resende CC, Araújo FAA, Sallenave RNUR. Análise epidemiológica dos acidentes ofídicos. Ministério da Saúde. Secretaria Nacional de Ações Básicas de Saúde. Brasília, 1989. 
17. Ribeiro LA. Epidemiology of ophidic accidents. Memórias do Instituto Butantan 52 (supl):15-16, 1990.

18. Ribeiro LA, Campos VAFP, Albuquerque M, Takaoka NY. Acidente ofídico no Estado de São Paulo. Revista da Associação Médica Brasileira 39:4-7, 1993.

19. Ribeiro LA, Jorge MT. Epidemiologia e quadro clínico dos acidentes por serpentes Bothrops jararaca adultas e filhotes. Revista do Instituto de Medicina Tropical de São Paulo 32:436-442, 1990.

20. Rosenfeld G. Symptomatology, pathology, and treatment of snakebite in South America. In: Bucherl W, Buckley EE (eds) Venomous animals and their venoms. Academic Press, New York, p.345-384, 1971.

21. Rosenfeld G. Animais peçonhentos e tóxicos do Brasil. In: Lacaz CS, Baruzzi RG, Siqueira E (eds) Introdução à geografia médica do Brasil. Ed. Edgard Blucher, São Paulo, p.430-475, 1972.

22. Secretaria de Saúde do Estado de São Paulo. Manual de vigilância epidemiológica; acidentes por animais peçonhentos; identificação, diagnóstico e tratamento. Centro de Vigilância Epidemiológica "Professor Alexandre Vranjac", Instituto Butantan. Secretaria de Estado da Saúde, São Paulo, 1993.

23. Sazima I. Um estudo de biologia comportamental da jararaca, Bothrops jararaca, com uso de marcas naturais. Memórias do Instituto Butantan 50:8399, 1988

24. Sazima I. Natural history of the jararaca pitviper, Bothrops jararaca, in Southeastern Brazil.
In: Campbell JA, Brodie ED (eds) Biology of the pitvipers. Selva Publ,Tyler,Texas, p.199-216, 1992.

25. Sgarbi LPS, Ilias M, Machado T, Alvarez L, Barravieira B. Human envenomations due to snakebites in Marilia, state of São Paulo, Brazil, a retrospective epidemiological study. The Journal of Venomous Animals and Toxins 1:70-78, 1995.

26 Silva CJ, Ribeiro LA, Silveira PVP, Nishioka SA, Jorge MT. Epidemiologia dos acidente ofídicos em Uberlândia, MG e municípios próximos. Dados preliminares. In: Resumos do XXXI Congresso da Sociedade Brasileira de Medicina Tropical, São Paulo p.10, 1995.

27. Takaoka NY, Albuquerque MJ, Campos VAFP, Gualtieri VB, Katz G, Jorge MT, Ribeiro LA. Distribuição dos acidentes por Bothrops, Crotalus e Micrurus segundo os Escritõrios Regionais de Saúde (ERSAS), SP, 1990/92. Revista da Sociedade Brasileira de Medicina Tropical 27(supl 1):118, 1994.

28. Teixeira R. Forma grave do acidente ofídico da sub-família crotalinae. Anais da Academia de Medicina da Bahia 2:109-137, 1979.

29. Torres JB, Carlotto PR. Levantamento dos gêneros de ofídios e espécies de aracnideos causadores de acidentes na casuística do centro de informações do Rio Grande do Sul. Memórias do Instituto Butantan 46:207-218, 1982.

30. Vêncio D, Oliveira DA. Ofidismo no Estado de Goiás. Revista Goiana de Medicina 26:125-130, 1980. 